\title{
LINKING HIPPARCOS TO GALAXIES: THE BONN AND POTSDAM PROGRAMMES
}

\author{
P. BROSCHE ${ }^{1}$, W. R. DICK ${ }^{1,2}$, R. GALAS $^{1}$, M. GEFFERT ${ }^{1}$, S. HIRTE ${ }^{2}$, \\ E. SCHILBACH ${ }^{2}$, R. - D. SCHOLZ ${ }^{2}$ \\ ${ }^{1}$ Sternwarte der Universität Bonn \\ Auf dem Hügel 71 \\ $D-W-5300$ Bonn 1 \\ F.R. Germany \\ ${ }^{2}$ Sternwarte Babelsberg \\ Rosa-Luxemburg-Str. $17 a$ \\ D-0-1591 Potsdam \\ F. R. Germany
}

Besides the Iink of the HIPPARCOS reference frame to extragalactic objects via radio stars or by the HST, also photographic astrometry is able to calibrate the HIPPARCOS proper motions with regard to an inertial system. Numerical simulations have shown that even with a very small number of well-distributed link fields ( 3 to 5 ) the photographic method is competitive with other techniques.

The Bonn programme is based on the determination of the fictitious proper motion of a quasar or a compact galaxy with respect to the reference stars in each field (Brosche 1980). From the fact that the true proper motions of the quasars are vanishingly small, conditions for the preliminary HIPPARCOS system can be obtained. Such conditions have been derived for 45 stars in the surrounding of four quasars from measurements on 65 plates with epoch differences of up to 90 years (Brosche et al. 1991). Measurements for a fifth field are in progress. Even with the data obtained so far it will be possible to achieve the link with random errors smaller than $0^{\prime \prime} .15$ per century.

The Potsdam/Tautenburg programme uses the plate vault of the Tautenburg Schmidt telescope containing about 70 potential proper motion fields with minimum epoch differences of 20 years. Absolute proper motions of stars from the HIPPARCOS Input Catalogue will be derived using faint and compact galaxies as a reference (Dick et al. 1987). Measurements have been completed for 19 fields. From the first reductions of these measurements it has been estimated that about one half of the HIPPARCOS stars in the fields can be used as link stars. With these data a link with random errors smaller than 0".1 per century should be possible (Yatsenko et al. 1987).

\section{References}

Brosche P., 1980, AJ 85, 1674

Brosche P., Ducourant C., Galas R., Geffert M., Karafistan A., 1991, $A \& A 245,669$

Dick W.R., Ruben G., Schilbach E., Scholz R.-D., 1987,

Astron. Nachr. 308, 211

Yatsenko A. I., Rybka S.P., Scholz R. -D., 1987, Astron. Nachr. 308, 319 